\title{
Acknowledgement to Reviewers of Lubricants in 2016
}

\author{
Lubricants Editorial Office \\ Published: 10 January 2017 \\ MDPI AG, St. Alban-Anlage 66, 4052 Basel, Switzerland; lubricants@mdpi.com
}

The editors of Lubricants would like to express their sincere gratitude to the following reviewers for assessing manuscripts in 2016.

We greatly appreciate the contribution of expert reviewers, which is crucial to the journal's editorial process. We aim to recognize reviewer contributions through several mechanisms, of which the annual publication of reviewer names is one. Reviewers receive a voucher entitling them to a discount on their next MDPI publication and can download a certificate of recognition directly from our submission system. Additionally, reviewers can sign up to the service Publons (https://publons.com) to receive recognition. Of course, in these initiatives we are careful not to compromise reviewer confidentiality. Many reviewers see their work as a voluntary and often unseen part of their role as researchers. We are grateful to the time reviewers donate to our journals and the contribution they make.

If you are interested in becoming a reviewer for Lubricants, see the link at the bottom of the webpage http://www.mdpi.com/reviewers.

The following reviewed for Lubricants in 2016:

$\begin{array}{ll}\text { Abd Rahim, Erween } & \text { Dimaki, Andrey } \\ \text { Adams, Michael } & \text { Dowson, Duncan } \\ \text { Affatato, Saverio } & \text { Etxeberria, Jon } \\ \text { Ali, W. Y. } & \text { Evaristo, Manuel } \\ \text { Antoszewski, Bogdan } & \text { Gao, Leiming } \\ \text { Atkin, Rob } & \text { Gara, Luan } \\ \text { Bal, B. Sonny } & \text { Gharam, A. Abou } \\ \text { Becerir, Behcet } & \text { González, Begoña } \\ \text { Bełdowski, Piotr } & \text { Gül, H. } \\ \text { Bermúdez, Maria } & \text { Gustavsson, Fredrik } \\ \text { Bexell, U. } & \text { Heim, Frederic } \\ \text { Bilisik, Kadir } & \text { Heise, Rainer } \\ \text { Blanco, David } & \text { Hu, Yuanzhong } \\ \text { Bobzin, Kirsten } & \text { Hülsmann, Michael } \\ \text { Brostow, Witold } & \text { Iglesias, Patricia } \\ \text { Campos, Armando } & \text { Izadi, Hossein } \\ \text { Celichowski, Grzegorz } & \text { Jimenez, Ana Eva } \\ \text { Chiñas-Castillo, Fernando } & \text { Kaneta, Motohiro } \\ \text { Choudhary, Dipankar } & \text { Khonsari, Michael } \\ \text { Colombo, Luigi } & \text { Kinoshita, Hiroshi } \\ \text { Cousseau, Tiago } & \text { Konieczny, J. } \\ \text { Crockett, Rowena } & \text { Kuhn, Erik } \\ \text { De Pellegrin, Dennis V. } & \text { Lewis, Roger } \\ \text { Demas, Nicholaos } & \text { Ma, Kung-Jeng } \\ \text { Demirci, Ibrahim } & \text { Maleque, Md Abdul } \\ \text { Dennington, Simon } & \text { Malucelli, Giulio } \\ & \end{array}$

MArcos Barcena, Mariano Maritinez-Mateo, Isidoro Maru, Marcia M. Matthews, Allan Mezghani, Sabeur Minami, Ichiro Miura, Yoshiko Morris, Nick Nallasamy, $\mathrm{P}$ Negrini, Daniella $\mathrm{Nie}$, Songlin Nurul, Mohd Ahyan Okabe, Kazunari Omrani, Emad Pagano, F Pantelis, Nikolakopoulos Pawlus, Pawel Perry, Scott S. Pettarin, Valeria Pisaturo, Mario Piwoński, Ireneusz Riahi, Mohammad Righi, Maria Clelia Rigney, David Rodriguez Ripoll, Manel Ruggiero, Alessandro 
Saikko, Vesa Salguero, Jorge

Santamaria Razo, Diego

Schmauder, Siegfried

Senatore, Adolfo

Shum, P.W.

Sonntag, Robert

Szewczyk, Roman
Totolin, Vladimir

Van Citters, Douglas W.

Vengudusamy, B

Vieira, Manuel Fernando

Wang, Shibo

Wang, Tingmei

Woschke, Elmar

Yin, Yaobao
Yoon, Moon-Young

Zak, Alla

Zekonyte, Jurgita

Zhang, Chao

Zhmud, Boris

Zhu, Jianjun

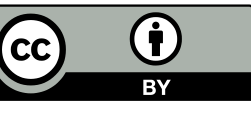

(C) 2017 by the authors; licensee MDPI, Basel, Switzerland. This article is an open access article distributed under the terms and conditions of the Creative Commons Attribution (CC-BY) license (http://creativecommons.org/licenses/by/4.0/). 